\title{
Fully tuneable, Purcell-enhanced solid-state quantum emitters
}

\author{
M. Petruzzella, ${ }^{1, a)}$ T. Xia, ${ }^{1}$ F. Pagliano, ${ }^{1}$ S. Birindelli, ${ }^{1}$ L. Midolo, ${ }^{2}$ Z. Zobenica, ${ }^{1}$ L. H. Li, ${ }^{3}$ \\ E. H. Linfield, ${ }^{3}$ and A. Fiore ${ }^{1}$ \\ ${ }^{1}$ COBRA Research Institute, Eindhoven University of Technology, P.O. Box 513, NL-5600MB Eindhoven, \\ The Netherlands \\ ${ }^{2}$ Niels Bohr Institute, University of Copenhagen, Blegdamsvej 17, DK-2100 Copenhagen, Denmark \\ ${ }^{3}$ School of Electronic and Electrical Engineering, University of Leeds, Leeds LS2 9JT, United Kingdom
}

(Received 25 June 2015; accepted 28 September 2015; published online 8 October 2015)

\begin{abstract}
We report the full energy control over a semiconductor cavity-emitter system, consisting of single Stark-tunable quantum dots embedded in mechanically reconfigurable photonic crystal membranes. A reversible wavelength tuning of the emitter over $7.5 \mathrm{~nm}$ as well as an $8.5 \mathrm{~nm}$ mode shift are realized on the same device. Harnessing these two electrical tuning mechanisms, a single exciton transition is brought on resonance with the cavity mode at several wavelengths, demonstrating a ten-fold enhancement of its spontaneous emission. These results open the way to bring several cavity-enhanced emitters mutually into resonance and therefore represent a key step towards scalable quantum photonic circuits featuring multiple sources of indistinguishable single photons. (C) 2015 AIP Publishing LLC. [http://dx.doi.org/10.1063/1.4932946]
\end{abstract}

Last decade has witnessed pioneering advancements in the development of the elementary building blocks for envisioned quantum photonic circuits, ${ }^{1}$ which may enable simulating problems, which are intractable on classical computers. $^{2,3}$ Efficient on-demand single-photon sources, obtained by coupling a quantum emitter to an optical cavity, represent one of these key building blocks. Additionally, cavity quantum electrodynamics (c-QED) offers a viable solution to create a coherent and efficient interface between light and matter qubits, as needed to establish entanglement between distant quantum emitters via a photonic channel. ${ }^{4}$ Among its numerous solid-state implementations, quantum dots (QDs) embedded in semiconductor nano-resonators have emerged as one of the most promising integrated platforms, ${ }^{5,6}$ specifically for the on-demand generation of single and entangled photons. ${ }^{7}$ Coupling to photonic crystal cavities (PCCs) is notably attractive due to their engineerable electromagnetic environment which provides record quality factors $(\mathrm{Q})$ in a wavelength-scale volume. ${ }^{8}$ Indeed, the basic c-QED phenomena have been recently demonstrated, including Rabi splitting, ${ }^{9}$ static $^{10}$ and dynamic ${ }^{11,12}$ control of spontaneous emission and single-photon non-linearities. ${ }^{13,14}$ Nevertheless, integrating and interconnecting multiple c-QED nodes within the same chip poses considerable scalability issues.

One of the leading experimental challenges in this context resides in the spectral matching of multiple cavityemitter systems, which requires the deterministic control over the energy of both emitters and cavities. To this end, post-processing tuning strategies are imperative because of the QD inhomogeneous broadening and the intrinsic fabrication imperfections which spread the actual cavity resonance over several nanometers.

Lately, a number of techniques based on electric, magnetic, temperature and strain control have been successfully employed to tune the emitters' energy. ${ }^{5}$ On the other hand,

${ }^{\text {a)} E l e c t r o n i c ~ m a i l: ~ m . p e t r u z z e l l a @ t u e . n l ~}$ several proposals have been adopted to tune the cavity spectrum, including thermal methods, ${ }^{15}$ gas adsorption, ${ }^{16}$ photochromic materials, ${ }^{17}$ photo-oxidation, ${ }^{18}$ free carrier injection, ${ }^{19,20}$ and nano-electromechanical systems. ${ }^{21-23}$

However, so far, the crucial goal of achieving a simultaneous energy control of an integrated emitter and its cavity has not been attained yet. In this letter we present a fully tuneable cavity-emitter system, where both the cavity and emitter wavelengths can be independently controlled in the same semiconductor device. This enables the demonstration of Purcell-enhancement from an energy-tuneable single dot.

Our device is sketched in Figure 1 and incorporates two parallel photonic crystal membranes which can be vertically displaced by capacitive forces. ${ }^{22}$ To this end, an n-i-p diode realized across the membranes provides the electrostatic actuation when it is operated in reverse bias. The resulting nano-mechanical displacement modifies the effective index of the coupled modes of the double-membrane waveguide, leading to a blue(red)-shift of the vertically anti-symmetric (symmetric) modes. ${ }^{24}$ The QD region is grown in the middle of the top slab to remove any interaction with the electrostatic field. ${ }^{23}$ A second p-i-n diode, sharing the n-layer with the cavity-tuning diode, is fabricated on the top membrane to apply a vertical electric field across the QD layer. In such a

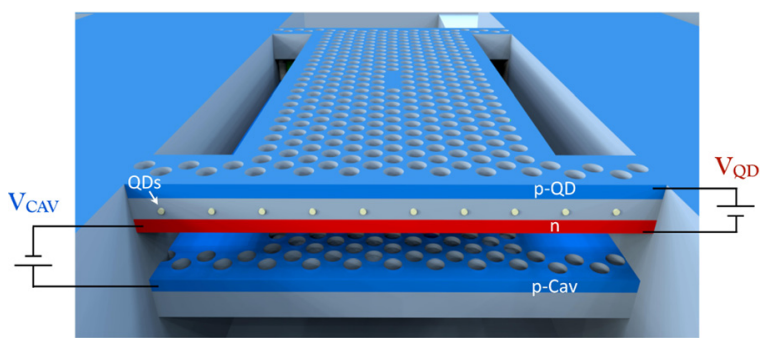

FIG. 1. (a) Sketch of the device illustrating the photonic crystal cavity patterned through two vertically coupled membranes. A top p-i-n diode realized across the top slab governs the QD energy via the quantum-confined Stark effect, while a second n-i-p diode controls the cavity resonance through capacitive forces. 
way, the emitter's energy is tuned by the quantum-confined Stark effect. ${ }^{25}$

The sample is grown by molecular beam epitaxy and includes two GaAs slabs of equal thickness $(170 \mathrm{~nm})$ isolated by a 240 -nm-thick sacrificial $\mathrm{Al}_{0.7} \mathrm{Ga}_{0.3}$ As spacer. A $1.5 \mu \mathrm{m}$ thick $\mathrm{Al}_{0.7} \mathrm{Ga}_{0.3}$ As layer separates the double membranes from the undoped (001) GaAs substrate. Low-density InAs QDs are grown in the middle of the upper membrane in the Stranski-Krastanov mode at a very low growth rate. ${ }^{26}$ Additionally, two 17-nm-thick $\mathrm{Al}_{0.3} \mathrm{Ga}_{0.7} \mathrm{As}$ barriers are introduced $8 \mathrm{~nm}$ above and below the QDs in order to suppress the tunneling probability of electron-hole pairs out of the dot and consequently increase the exciton tuning range. ${ }^{27}$ The upper 50-nm thick region of both membranes is p-doped whereas the bottom 50 -nm-thick part of the top slab is n-doped $\left(p_{Q D}=1.5 \times 10^{18}, n=p_{\text {cav }}=2 \times 10^{18} \mathrm{~cm}^{-3}\right)$. The fabrication consists in the realization of the double diodes followed by the patterning of the photonic crystal structure. Employing two optical lithographic steps and selective wet etchings, vias to the $\mathrm{p}$ and $\mathrm{n}$ regions are opened and $\mathrm{Ti} / \mathrm{Au}$ $(50 / 200 \mathrm{~nm})$ pads are evaporated on top of them. In this way, two $\mathrm{p}$-i-n diodes sharing a common n-layer are realized in a single lift-off process. Together with the p-via, a flexible four-arm micro-bridge is also defined on the top membrane. A PCC created by three missing holes (lattice constant $\mathrm{a}=395 \mathrm{~nm}$ and radius $r=0.31 a$ ) is defined on a 400-nmthick $\mathrm{Si}_{3} \mathrm{~N}_{4}$ mask by $30 \mathrm{kV}$-electron beam lithography and a reactive ion etching step. The radii and positions of the ten holes surrounding the defect region are modified to provide a theoretical quality factor $\mathrm{Q}=34000$, as calculated by $3 \mathrm{D}$ finite-element modeling. Subsequently, the PCC is vertically transferred to both membranes by inductively coupled plasma etching. The release of the free standing structure is carried out by selectively removing the sacrificial layers via cold $\left(1^{\circ} \mathrm{C}\right) \mathrm{HCl}$ solution while keeping the $\mathrm{Si}_{3} \mathrm{~N}_{4}$ mask to avoid sticking arising from capillary forces. ${ }^{22}$ Finally, the $\mathrm{Si}_{3} \mathrm{~N}_{4}$ layer is eliminated by isotropic $\mathrm{O}_{2}-\mathrm{CF}_{4}$ plasma ashing.
Figure 2 shows the scanning electron micrographs of the full device.

Low-temperature $(\mathrm{T}=9 \mathrm{~K})$ micro-photoluminescence $(\mu \mathrm{PL})$ experiments were performed in a continuous-flow helium cryostat equipped with two electrical probes sharing a common ground and approaching the sample from opposite directions. QDs were excited with an above-bandgap diode laser $(\lambda=785 \mathrm{~nm})$ and their emission was collected through the same objective (numerical aperture NA $=0.4$ ) and analyzed by a fiber-coupled spectrometer, after being spectrally isolated from the pump laser via a dichroic beam-splitter.

Figure 3(a) shows the color-coded $\mu \mathrm{PL}$ measurements acquired in the strong pumping regime $(50 \mu \mathrm{W}$ measured before the objective) in order to probe the cavity modes while sweeping the voltage across the membranes. The spectrum is dominated by four eigenmodes of the PCC cavity. By increasing the reverse bias across the membranes $\left(V_{\mathrm{CAV}}\right)$, the two pairs of modes shift in wavelengths along opposite directions. This effect represents the unambiguous signature of the mechanical origin of the mode-tuning and rules out any possible thermal origin.

Here, a maximum and reproducible cavity shift of $8.5 \mathrm{~nm}$ is achieved at $-4.5 \mathrm{~V}$, without reaching the irreversible pull-in condition that brings the structure to collapse. In addition, from the tuning direction, we classify the highenergy modes as anti-symmetric $\left(C_{A S 1}, C_{A S 2}\right)$ and two modes situated around $1330 \mathrm{~nm}$ as symmetric $\left(C_{S Y 1}, C_{S Y 2}\right)$. From a comparison with the spectrum calculated using a finiteelement method, we attributed the anti-symmetric modes to lowest-order modes mainly polarized perpendicular to the cavity axis, while the symmetric lines have been identified as higher order-modes with dominant polarization along the cavity axis. The quality factor of these modes is around 1000 , while quality factors up to 5000 have been measured on other devices on the same chip, showing that $\mathrm{Q}$ factors adequate for the enhancement of spontaneous emission are possible in the double-membrane structure.
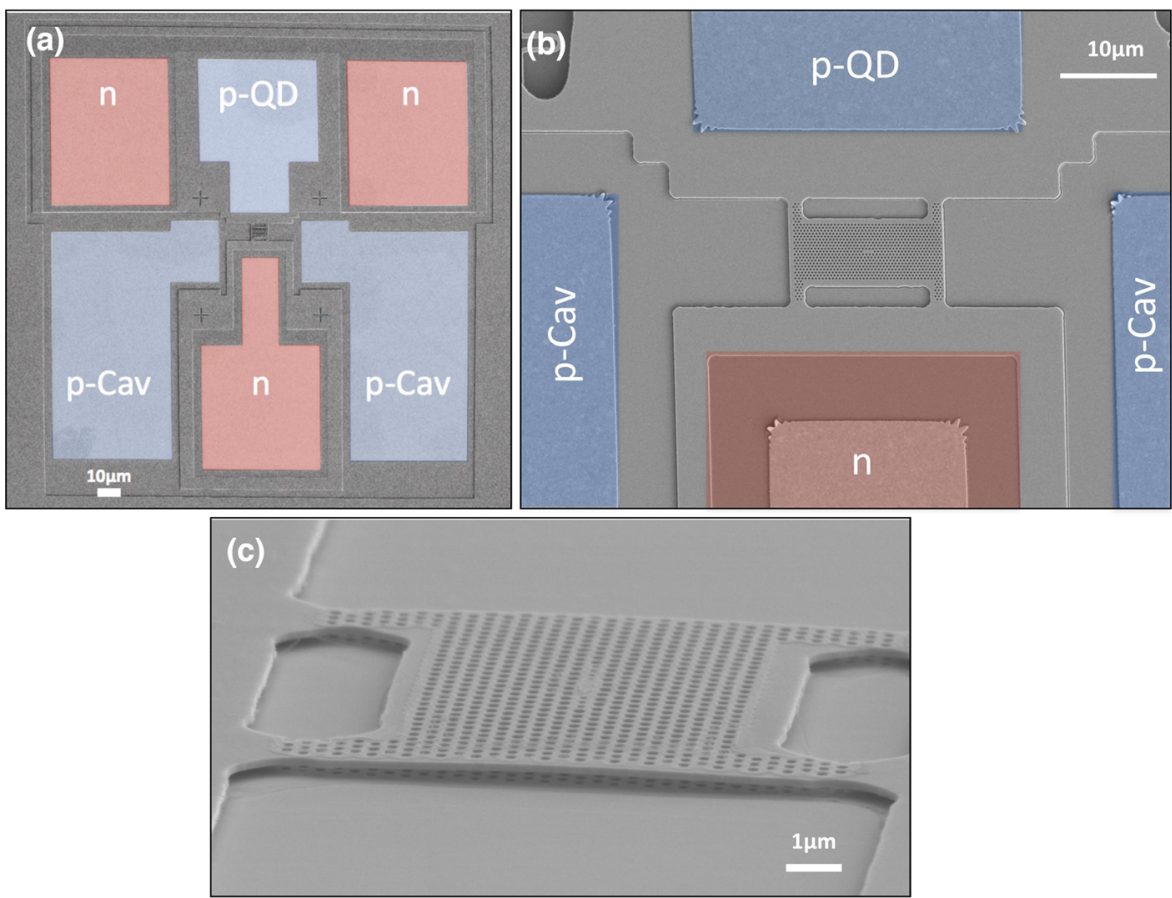

FIG. 2. Scanning electron micrographs (SEMs) of the device showing the contact mesa structure of the double diodes (a) and a zoom (b) into a typical PCC realized on a four-arm $12-\mu \mathrm{m}$ long bridge. (c) Tilted SEM image of the device used in the experiments. The cavity is realized by removing three holes from the lattice and modifying the radii and relative positions of the six in-line and four vertical holes surrounding the cavity-defect. 

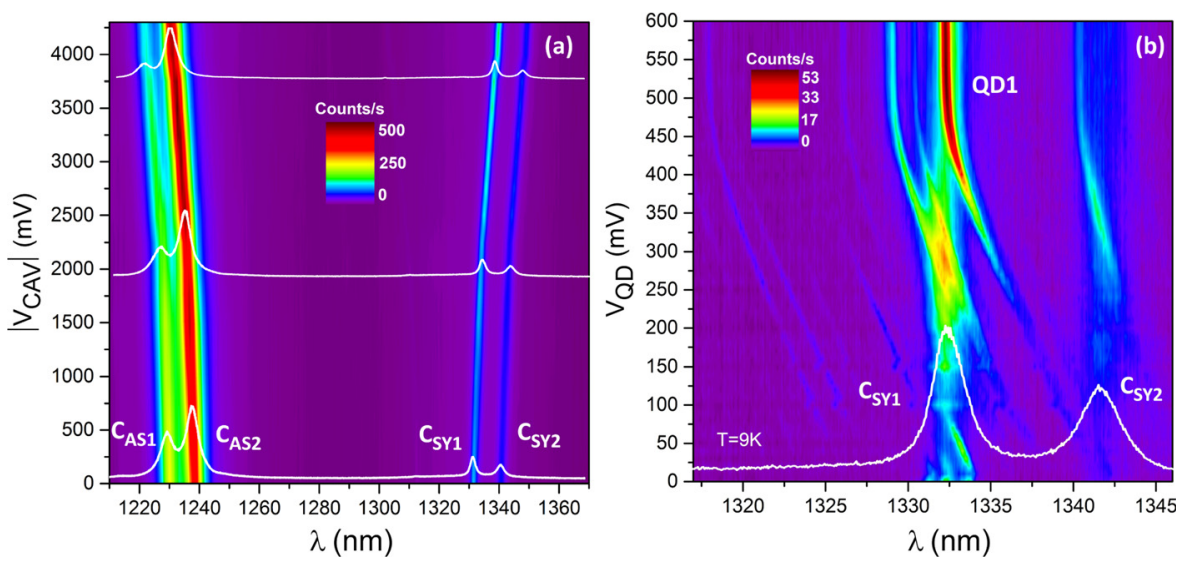

In the following tuning experiments, we focus on the symmetric mode $C_{S Y 1}$, which is located around the ground state of the QDs. When this mode is shifted in frequency, a modulation of its quality factor is observed ranging from 620 to 900 . The oscillation in the cavity losses can be related to the change in the amount of wave-vector components of the cavity field lying inside the light cone when the two slabs are brought closer. ${ }^{28}$

After reducing the laser power to the $\mathrm{nW}$-level $(80 \mathrm{nW})$, just below the saturation level of QDs, also single QD lines appear in the spectrum. Figure 3(b) presents the QD tuning experiments carried out on the same device. Here, the colorcoded image is built up from several $\mu$-PL spectra acquired while the Stark diode is operated in forward bias. When the voltage applied to the QD-diode is increased (in forward bias) from 0 to $0.6 \mathrm{~V}$, the exciton transitions shift to the blue achieving a maximum tuning range of $7.5 \mathrm{~nm}$. A clear enhancement of the spontaneous emission of single dot lines is observed when their energy crosses the cavity mode position. Remarkably, cavity modes are still visible in the configuration where there is no QD matching their energy due to the pumping from multi-excitons transitions ${ }^{29,30}$ and phonon-assisted feeding mechanisms. ${ }^{31}$ At negative bias the QD emission is suppressed suggesting that the built-in junction field $\left(F_{b i}=-200 \mathrm{KV} / \mathrm{cm}\right)$ is sufficient to sweep the carriers away from the active region. Besides, we observe the inhibition of the tuning close to the turn-on voltage of the diode $(0.6 \mathrm{~V})$ due to the increasing current. Consistently with previous reports on the quantum-confined Stark effect in InAs QDs, ${ }^{25}$ the blue shifting of single dot lines indicates that the electron wave function is located below the hole wave function with respect to the growth axis, giving rise to an inverted hole-electron alignment. From the fitting of the tuning curves, we estimated for the excitonic line QD1 a permanent dipole moment $p=-0.15 \pm 0.04 e \mathrm{~nm}$ and a polarizability $\beta=-4.9 \pm 0.1 \times 10^{-3} e \mathrm{~nm} \mathrm{kV}^{-1} \mathrm{~cm}$, comparable with previous works. ${ }^{12}$ The tuning rate is particularly high $\left(14.4 \frac{\mathrm{meV}}{V}\right)$ due to the thin intrinsic region $(70 \mathrm{~nm})$ of the Stark-diode.

The simultaneous control over the full emitter-cavity system is reported in Figure 4. Here, a quantum dot line (QD1) is first spectrally positioned at $\lambda_{1}=1332.2 \mathrm{~nm}$ by applying a Stark voltage of $V_{\mathrm{QD}}=590 \mathrm{mV}$. In this situation, resonance with the cavity mode is obtained at $V_{\mathrm{CAV}}=0 \mathrm{~V}$ (Figure 4(a)). By changing the Stark field $\left(V_{\mathrm{QD}}=320 \mathrm{mV}\right)$ the QD is red-shifted at a second wavelength $\lambda_{2}=1334.5 \mathrm{~nm}$ (Figure 4(b)). The cavity can be brought again into resonance at a voltage $V_{\mathrm{CAV}}=-1.4 \mathrm{~V}$ (Figure 4(b)). As shown in Figure 4(c), the electrical control on the exciton line does not significantly affect the cavity mode position (Figure 4(c)), despite the presence of an electro-optic effect in GaAs (the calculated electro-optic wavelength shift over the voltage range of Figure 4(c) is $0.01 \mathrm{~nm}$ ). However, when the cavity-diode is tuned from 0 to $1.6 \mathrm{~V}$ a crosstalk is observed on the QD-diode which results in a small blue-shift of the excitonic line QD1 by $0.4 \mathrm{~nm}\left(V_{\mathrm{QD}}=320 \mathrm{mV}\right)$ as reported in Figure $4(\mathrm{~d})$, whose origin is not understood. Additionally for $\left|V_{\mathrm{CAV}}\right| \cong 2.0 \mathrm{~V}$, the mode tuning rate decreases and the QDs lines start redshifting. We attribute this behavior to the increasing current flowing across the diodes. For a reverse cavity bias voltage $\left|V_{\mathrm{CAV}}\right|>2.0 \mathrm{~V}$ the current in the cavity diode was observed to strongly increase in the presence of a forward QD bias, which is indicative of the expected transistor behavior in this pinip junction. The corresponding temperature increase produces the red-shift of the QD lines observed in Figure 4(d). Notwithstanding, for a range of energies spanning over $2.6 \mathrm{meV}$ the wavelength-shift of QD lines is one order of magnitude less than the cavity tuning. This greatly facilitates the tuning of the entire quantum node from $\lambda_{1}$ to $\lambda_{2}$.

To further explore the QD-cavity coupling we investigated the temporal dynamics of the excitonic line QD1 in the weak pumping regime. ${ }^{32}$ Time-resolved experiments are performed employing an $80 \mathrm{MHz}$ pulsed diode laser $(\lambda=757 \mathrm{~nm}$, average power $\mathrm{P}=30 \mathrm{nW}$, pulse width $=70 \mathrm{ps})$ as excitation source, sending the PL emission through a tunable band-pass filter (bandwidth $=0.5 \mathrm{~nm}$ ) and making use of a superconducting single photon detector and a correlation card (PicoHarp300) to measure the photon arrival time. The full width at half maximum (FWHM) of the instrument response function (IRF) gives a temporal resolution of $90 \mathrm{ps.}$

The decay curves of the excitonic transition QD1 are reported in Figure 5. When the quantum dot line is spectrally aligned with the cavity resonance at $\lambda_{1}=1332.2 \mathrm{~nm}$ (purple dots, $\left.V_{\mathrm{QD}}=590 \mathrm{mV}, V_{\mathrm{CAV}}=0 \mathrm{mV}\right)$ and at $\lambda_{2}^{\prime}=1335.0 \mathrm{~nm}$ (red dots, $V_{\mathrm{QD}}=300 \mathrm{mV}, V_{\mathrm{CAV}}=-1600 \mathrm{mV}$ ), a decrease of its radiative lifetime is visible compared to the case in which the dot is set at $\lambda_{1}$, off-resonance from the cavitymode at $\lambda_{2}^{\prime}$ (blue dots).

From a bi-exponential fit convoluted with the IRF we derive the on-resonance time constants of the fast decay 


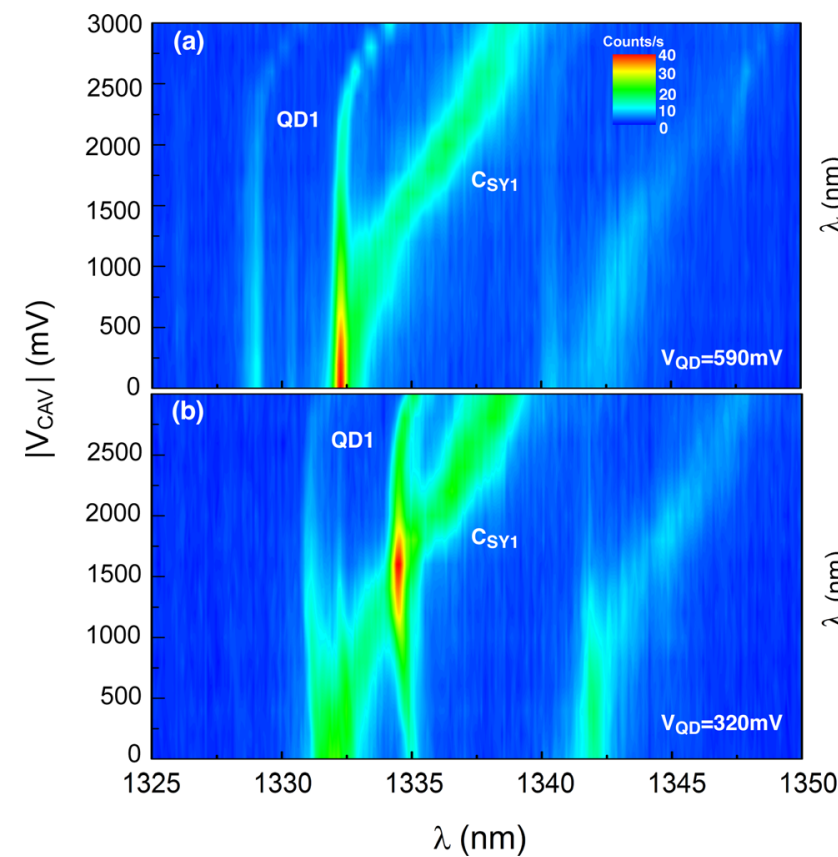

components related to cavity-enhanced single-QD emission $\tau_{\mathrm{ON}}\left(\lambda_{1}\right)=140 \mathrm{ps}, \tau_{\mathrm{ON}}\left(\lambda_{2}^{\prime}\right)=190 \mathrm{ps}$. The slow decay component $\tau_{\mathrm{ON} \text {.Slow }}\left(\lambda_{1}\right)=960 \mathrm{ps}$ is attributed to the residual contribution of the background-pumped cavity mode emission, which has a measured time constant $\tau_{\text {Mode }}=830 \mathrm{ps}$. The experimental error bar is estimated as $\approx 20$ ps from the standard deviation of values fitted from different measurements. In the off-resonance configuration the dot is characterized by a single exponential dynamics with time constant $\tau_{\mathrm{OFF}}=1.02 \pm 0.02 \mathrm{~ns}$. The QD ensemble data set is shown for comparison (green dots) and has a single decay constant $\tau_{\text {Bulk }}=1.55 \pm 0.02 \mathrm{~ns}$, similar to previous reports from similar QDs. ${ }^{33}$

In general the actual decay rate $\Gamma$ of a QD exciton in a $\mathrm{PCC}$ environment in the presence of an electric field can be

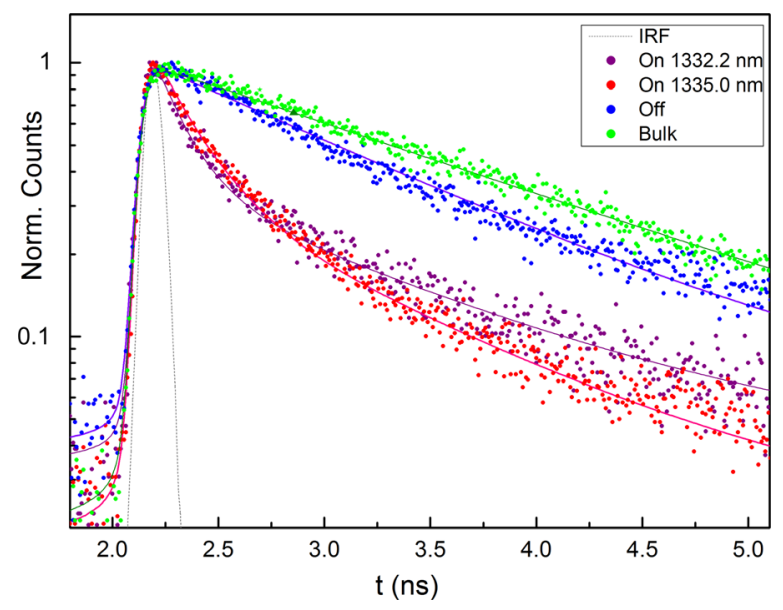

FIG. 5. Time-resolved normalized photoluminescence dynamics of the excitonic transition QD1 on resonance with the cavity mode at two distinct wavelengths, $\lambda_{1}=1332.2 \mathrm{~nm}$ (purple dots) and $\lambda_{2}^{\prime}=1335 \mathrm{~nm}$ (red dots). The off-resonance decay histogram of the dot positioned at $\lambda_{1}$, while the cavity wavelength is set at $\lambda_{2}^{\prime}$, is shown in blue. The QD ensemble evolution is reported in green as reference. Single and double exponential fits are shown with a continuous line and include the convolution with the IRF of the system (gray dashed line). written as ${ }^{34} \Gamma=\Gamma_{\mathrm{CAV}}+\Gamma_{\text {Leaky }}+\Gamma_{\mathrm{nr}}(F)$, where $\Gamma_{\mathrm{CAV}}$ represents the Purcell-enhanced emission in the cavity mode, $\Gamma_{\text {Leaky }}$ encodes the decay rate into the leaky modes of the PCC, while $\Gamma_{\mathrm{nr}}$ accounts for the non-radiative processes including tunneling of the electron out of the dot and depends on the applied electric field. However, within the low-field values used in this experiment $\left(F\left(\lambda_{1}\right)=-135 \mathrm{kV} /\right.$ $\mathrm{cm}$ and $\left.F\left(\lambda_{2}^{\prime}\right)=-167 \mathrm{kV} / \mathrm{cm}\right)$, both $\Gamma_{\mathrm{nr}}$ and the possible modification of the oscillator strength are small. ${ }^{27}$ Therefore, neglecting the decay channels not related to the presence of the cavity $\left(\Gamma_{\text {Leaky }}+\Gamma_{\mathrm{nr}}\right)$, estimated in the $(5-6 \mathrm{~ns})^{-1}$ range from literature data of similar QD heterostructure, ${ }^{35}$ we attribute the shortening of the on-resonance decay rate to the Purcell effect, ${ }^{36,37}$ quantifying a Purcell enhancement of $F_{P}\left(\lambda_{2}^{\prime}\right)=\frac{\tau_{O N}\left(\lambda_{2}^{\prime}\right)}{\tau_{\text {Bulk }}}=8 \pm 1$ and $F_{P}\left(\lambda_{1}\right)=\frac{\tau_{O N}\left(\lambda_{1}\right)}{\tau_{\text {Bulk }}}=11 \pm 2$ for the two wavelengths. The difference in the spontaneous emission enhancement at different wavelengths can be attributed to the modification of the quality factor when the cavity is tuned. Indeed, considering negligible the variation of the effective mode volume of the cavity (below $2 \%$ from simulations) the ratio between $Q\left(\lambda_{1}\right)=820 \pm 30$ and $Q\left(\lambda_{2}^{\prime}\right)=650 \pm 30$ is comparable to the ratio of the onresonance decay times $\frac{\tau_{O N}\left(\lambda_{1}\right)}{\tau_{O N}\left(\lambda_{2}^{\prime}\right)}=1.35 \pm 0.34$. We also note that the observed decrease in decay time when tuning the QD-cavity system from $\lambda_{2}^{\prime}$ to $\lambda_{1}$ cannot be explained by an increase in the tunneling rate, since the electric field applied for $\lambda_{1}$ is lower than the one for $\lambda_{2}^{\prime}$.

The suppression of the spontaneous emission offresonance is hampered by the limited tuning range in this experiment as confirmed by the ratio $\frac{\Gamma\left(\lambda_{\mathrm{CAV}}=\lambda_{\mathrm{QD}}=\lambda_{1}\right)}{\Gamma\left(\lambda_{\mathrm{CAV}}=\lambda_{2}^{\prime}, \lambda_{\mathrm{QD}}=\lambda_{1}\right)}=$ $10 \pm 3$ estimated from the spectral mismatch $\lambda_{1}-\lambda_{2}^{\prime}=2.8 \mathrm{~nm}$ using the expression in Ref. 38 and taking into account the emission into leaky modes and non-radiative recombination processes.

In conclusion, we have experimentally demonstrated a solid-state cavity-emitter system where both the energy of 
the emitter and the cavity resonance are independently and electrically controlled. By combining the Stark tuning of quantum dots with the nano-electromechanical actuation of the cavity the deterministic, reversible and real-time spectral alignment of the entire quantum node has been demonstrated. We studied the regime where the spontaneous emission of single excitons can be modulated by one order of magnitude at several energies. The integration of this platform with ridge waveguides, ${ }^{39}$ needed to transfer photons with low losses on the chip, will open the way to multi-node integrated cavity-quantum electrodynamics experiments and therefore de facto to scalable quantum sources for integrated quantum photonics.

We acknowledge stimulating discussions with R. W. van der Heijden, S. Fattah poor, and C. Jin. This research was financially supported by the Dutch Technology Foundation STW, Applied Science Division of NWO, the Technology Program of the Ministry of Economic Affairs under Project Nos. 10380 and 12662 and by NanoNextNL, a micro and nanotechnology program of the Dutch Ministry of Economic Affairs, Agriculture and Innovation (EL\&I) and 130 partners.

${ }^{1}$ J. L. O’Brien, A. Furusawa, and J. Vučković, Nat. Photonics 3, 687 (2009).

${ }^{2}$ A. Aspuru-Guzik and P. Walther, Nat. Phys. 8, 285 (2012).

${ }^{3} \mathrm{~S}$. Aaronson and A. Arkhipov, in Proceedings of the forty-third Annual ACM Symposium on Theory of Computing (ACM, 2011), pp. 333-342.

${ }^{4}$ H. Kimble, Nature 453, 1023 (2008).

${ }^{5}$ S. Buckley, K. Rivoire, and J. Vučković, Rep. Prog. Phys. 75, 126503 (2012).

${ }^{6}$ P. Lodahl, S. Mahmoodian, and S. Stobbe, Rev. Mod. Phys. 87, 347 (2015).

${ }^{7}$ A. J. Shields, Nat. Photonics 1, 215 (2007).

${ }^{8}$ Y. Akahane, T. Asano, B.-S. Song, and S. Noda, Nature 425, 944 (2003).

${ }^{9}$ T. Yoshie, A. Scherer, J. Hendrickson, G. Khitrova, H. Gibbs, G. Rupper, C. Ell, O. Shchekin, and D. Deppe, Nature 432, 200 (2004).

${ }^{10}$ P. Lodahl, A. F. Van Driel, I. S. Nikolaev, A. Irman, K. Overgaag, D. Vanmaekelbergh, and W. L. Vos, Nature 430, 654 (2004).

${ }^{11}$ C.-Y. Jin, R. Johne, M. Y. Swinkels, T. B. Hoang, L. Midolo, P. J. van Veldhoven, and A. Fiore, Nat. Nanotechnol. 9, 886 (2014).

${ }^{12}$ F. Pagliano, Y. Cho, T. Xia, F. van Otten, R. Johne, and A. Fiore, Nat. Commun. 5, 5786 (2014).

${ }^{13}$ D. Englund, A. Faraon, I. Fushman, N. Stoltz, P. Petroff, and J. Vučković, Nature 450, 857 (2007).

${ }^{14}$ I. Fushman, D. Englund, A. Faraon, N. Stoltz, P. Petroff, and J. Vučković, Science 320, 769 (2008).

${ }^{15}$ A. Faraon and J. Vučković, Appl. Phys. Lett. 95, 043102 (2009).
${ }^{16}$ S. Mosor, J. Hendrickson, B. Richards, J. Sweet, G. Khitrova, H. Gibbs, T. Yoshie, A. Scherer, O. Shchekin, and D. Deppe, Appl. Phys. Lett. 87, 141105 (2005).

${ }^{17}$ T. Cai, R. Bose, G. S. Solomon, and E. Waks, Appl. Phys. Lett. 102, 141118 (2013).

${ }^{18}$ A. Y. Piggott, K. G. Lagoudakis, T. Sarmiento, M. Bajcsy, G. Shambat, and J. Vučković, Opt. Express 22, 15017 (2014).

${ }^{19}$ F. Raineri, C. Cojocaru, R. Raj, P. Monnier, A. Levenson, C. Seassal, X. Letartre, and P. Viktorovitch, Opt. Lett. 30, 64 (2005).

${ }^{20}$ I. Fushman, E. Waks, D. Englund, N. Stoltz, P. Petroff, and J. Vučković, Appl. Phys. Lett. 90, 091118 (2007).

${ }^{21}$ M. Winger, T. Blasius, T. Mayer Alegre, A. H. Safavi-Naeini, S. Meenehan, J. Cohen, S. Stobbe, and O. Painter, Opt. Express 19, 24905 (2011).

${ }^{22}$ L. Midolo, P. van Veldhoven, M. Dundar, R. Notzel, and A. Fiore, Appl. Phys. Lett. 98, 211120 (2011).

${ }^{23}$ L. Midolo, F. Pagliano, T. Hoang, T. Xia, F. van Otten, L. Li, E. Linfield, M. Lermer, S. Höfling, and A. Fiore, Appl. Phys. Lett. 101, 091106 (2012).

${ }^{24}$ M. Notomi, H. Taniyama, S. Mitsugi, and E. Kuramochi, Phys. Rev. Lett. 97, 023903 (2006).

${ }^{25}$ P. Fry, I. Itskevich, D. Mowbray, M. Skolnick, J. Finley, J. Barker, E. O'Reilly, L. Wilson, I. Larkin, and P. Maksym, Phys. Rev. Lett. 84, 733 (2000).

${ }^{26}$ B. Alloing, C. Zinoni, V. Zwiller, L. H. Li, C. Monat, M. Gobet, G. Buchs, A. Fiore, E. Pelucchi, and E. Kapon, Appl. Phys. Lett. 86, 101908 (2005).

${ }^{27}$ A. J. Bennett, R. B. Patel, J. Skiba-Szymanska, C. A. Nicoll, I. Farrer, D. A. Ritchie, and A. J. Shields, Appl. Phys. Lett. 97, 031104 (2010).

${ }^{28}$ L. Midolo and A. Fiore, IEEE J. Quantum Electron. 50, 404 (2014).

${ }^{29}$ N. Chauvin, C. Zinoni, M. Francardi, A. Ino, L. Balet, B. Alloing, L. H. Li, and A. Fiore, Phys. Rev. B 80, 241306 (2009).

${ }^{30}$ M. Winger, T. Volz, G. Tarel, S. Portolan, A. Badolato, K. J. Hennessy, E. L. Hu, A. Beveratos, J. Finley, and V. Savona, Phys. Rev. Lett. 103, 207403 (2009).

${ }^{31}$ U. Hohenester, A. Laucht, M. Kaniber, N. Hauke, A. Neumann, A. Mohtashami, M. Seliger, M. Bichler, and J. J. Finley, Phys. Rev. B 80, 201311 (2009).

${ }^{32}$ E. Moreau, I. Robert, J. Gérard, I. Abram, L. Manin, and V. Thierry-Mieg, Appl. Phys. Lett. 79, 2865 (2001).

${ }^{33}$ L. Balet, M. Francardi, A. Gerardino, N. Chauvin, B. Alloing, C. Zinoni, C. Monat, L. Li, N. Le Thomas, and R. Houdré, Appl. Phys. Lett. 91, 123115 (2007).

${ }^{34}$ A. Laucht, F. Hofbauer, N. Hauke, J. Angele, S. Stobbe, M. Kaniber, G. Böhm, P. Lodahl, M. Amann, and J. Finley, New J. Phys. 11, 023034 (2009).

${ }^{35}$ T. B. Hoang, J. Beetz, M. Lermer, L. Midolo, M. Kamp, S. Höfling, and A. Fiore, Opt. Express 20, 21758 (2012).

${ }^{36}$ E. Purcell, Phys. Rev. 69, 37 (1946).

${ }^{37}$ J. Gérard, B. Sermage, B. Gayral, B. Legrand, E. Costard, and V. ThierryMieg, Phys. Rev. Lett. 81, 1110 (1998).

${ }^{38}$ D. Englund, D. Fattal, E. Waks, G. Solomon, B. Zhang, T. Nakaoka, Y. Arakawa, Y. Yamamoto, and J. Vučković, Phys. Rev. Lett. 95, 013904 (2005).

${ }^{39}$ S. Fattah poor, T. Hoang, L. Midolo, C. Dietrich, L. Li, E. Linfield, J. Schouwenberg, T. Xia, F. Pagliano, F. van Otten, and A. Fiore, Appl. Phys. Lett. 102, 131105 (2013). 